\title{
THE NONVANISHING OF CERTAIN CHARACTER SUMS
}

\author{
S. ULLOM ${ }^{1}$
}

ABSTRACT. Let $\boldsymbol{x}$ be a Dirichlet character with conductor $f$ and $M(\mathbf{x})=\Sigma a \overline{\mathbf{x}}(a)$, summation over integers $a$ prime to $f$ and $1 \leq a<f$. It is well known that the nonvanishing of the Dirichlet $L$-function $L(s, \boldsymbol{x})$ at $s=1$ implies $M(\boldsymbol{x}) \neq 0$ for $\boldsymbol{x}$ imaginary, i.e. $\boldsymbol{x}(-1)=-1$. This article provides a purely algebraic proof that $M(x) \neq 0$ when the conductor $f$ is a prime power and the imaginary $x$ is either a faithful character or has order a power of 2 .

1. Introduction. Let $\chi$ be a Dirichlet character with conductor $f$. If $\chi$ is imaginary, that is $\chi(-1)=-1$, then the character sum $M(\chi) \neq 0$, where $M(\chi)=\Sigma_{a} a \bar{\chi}(a)$, summation over integers $a$ prime to $f$ and $1 \leq a<f$. In fact one knows $[1, \S 4]$ that the Dirichlet $L$-function $L(s, \chi)$ at $s=1$ is

$$
L(1, \chi)=f^{-2} \pi i r(\chi) M(\chi), \quad \tau(\chi) \text { a Gauss sum, }
$$

and $L(1, \chi) \neq 0$.

The problem is to find an elementary proof that $M(\chi) \neq 0$. Hasse $[1$, $\S \S 31-32]$ obtained congruence properties of $M(\chi)$ which imply $M(\chi) \neq 0$ when the conductor $f$ is a prime power and $\chi$ is either a faithful character or has order a power of 2. The purpose of this article is to give another algebraic proof of this result. The calculations of our method take place in a certain integral group ring and involve elements $\alpha_{\phi}$ which are essentially idempotents in the rational group ring. There is still no algebraic proof that $M(\chi) \neq 0$ for all imaginary $\chi$.

2. Preliminaries. In this article we fix $f, K=Q(\sqrt[f]{1})$, and the Galois group $G=G(K / Q)$. The group $G$ is isomorphic to $(Z / f Z)^{\times}$by $s_{a} \rightarrow a \bmod f$,

Received by the editors September 7, 1973.

AMS (MOS) subject classifications (1970). Primary 10G05, 12A35; Secondary 16A26, $12 \mathrm{~A} 50$.

Key words and phrases. Character sum, cyclotomic field, class number, integral group ring.

1 This research was partially supported by a contract with the National Science Foundation. 
$(a, f)=1$; here $s_{a} \in G$ raises an $f$ th root of 1 to its ath power. The semisimple $Q$-algebra $Q G$ is isomorphic to a direct sum $\Sigma F_{\phi}$ of cyclotomic fields $F_{\phi}$, which correspond to the irreducible rational characters (in the sense of representation theory) of $Q G$. Each homomorphism $\chi: G \rightarrow C^{\times}, C$ complex numbers, determines a rational character $\phi$ as follows. Let $Q(\chi)$ be the field of values of $\chi$, then $\phi(s)=\Sigma_{\gamma} \gamma(\chi(s))$, s $\in G$, summation over $\gamma \in G(Q(\chi) / Q)$; the field $F_{\phi}=Q(\chi)$. Denote by $e_{\phi}$ the primitive idempotent of $Q G$

$$
e_{\phi}=\frac{1}{|G|} \sum_{s \in G} \phi\left(s^{-1}\right) s .
$$

Then $\chi$ extended to $Q G$ defines an isomorphism $\chi: Q G e_{\phi} \rightarrow F_{\phi}$. Define

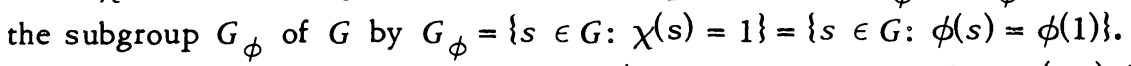
Choose $s_{\phi} \in G$ generating the group $G / G_{\phi}$ of order say $g$; then $\chi\left(s_{\phi}\right)$ is a primitive gth root of 1 .

Let $\omega=\Sigma_{a} a s_{a}^{-1},(a, f)=1,1 \leq a<f$. Then $\chi(\omega)=M(\chi)$. Thus $M(\chi) \neq 0$ for all imaginary characters $\chi$ of $G$ iff $\omega e^{-}\left(e^{-}=1 / 2\left(1-s_{-1}\right)\right)$ is not a zero divisor of $Q G e^{-}$.

3. Group ring elements $\alpha_{\phi}$. For each irreducible rational character $\phi$ of $G$ define the nonzero element $\alpha_{\phi}$ of $Z G$ by

$$
a_{\phi}=\sigma(\phi) \prod_{q}\left(1-s_{\phi}^{g / q}\right) \text {. }
$$

Here $\sigma(\phi)$ is the sum of the elements of $G_{\phi}$ and the product is taken over all primes $q$ dividing $g$.

(3.1) Lemma. Let $\phi$ and $\psi$ be irreducible rational characters of $Q G$. The product of $\alpha_{\phi}$ with $e_{\psi}$ is

$$
\begin{aligned}
\alpha_{\phi} e_{\psi} & =\left.\right|_{\phi} \mid \prod_{q}\left(e_{\phi}-\left(s_{\phi} e_{\phi}\right)^{g / q}\right) & & \text { if } \phi=\psi, \\
& =0 & & \text { if } \phi \neq \psi .
\end{aligned}
$$

Thus, if the complex character $\chi$ determines $\phi, M(\chi)=0$ iff $\omega e_{\phi}=0$ iff $\omega \alpha_{\phi}=0$.

Proof. Suppose $G_{\phi} \subset G_{\psi}$. Since $t \in G_{\psi}$ implies $t e_{\psi}=e_{\psi}$, we have

$$
a_{\phi} e_{\psi}=\left|G_{\phi}\right| \prod_{q}\left(e_{\psi}-\left(s_{\phi} e_{\psi}\right) g / q\right) .
$$

If $G_{\phi}=G_{\psi}$ (so $\left.\phi=\psi\right)$, we obtain the desired result. If $G_{\phi}$ is a proper subgroup of $G_{\psi}$, there exists a prime $q$ dividing $g$ such that $s_{\phi}^{g / q} \in G_{\psi}$. For such $q$, the factor $e_{\psi}-\left(s_{\phi} e_{\psi}\right)^{g / q}=0$. 
On the other hand, suppose $G_{\phi}$ is not contained in $G_{\psi}$. Then the order of $G_{\phi^{\prime}} / G_{\phi} \cap G_{\psi}$ is $h>1$. Choose $t \in G_{\phi}$ generating $G_{\phi^{\prime}} / G_{\phi} \cap G_{\psi}$. Since $b>1, \sum_{i=1}^{b} t^{i} e_{\psi}=0$ and then $\sigma(\phi) e_{\psi}=0$. Thus $a_{\phi} e_{\psi}=0$ if $\phi \neq \psi$. The last assertion of $(3.1)$ is now clear.

(3.2) Proposition. Suppose $f=p^{r}$, p prime. If $\chi$ is a nontrivial faithful character (i.e. $G_{\phi}=1$ ) of $(Z / f Z)^{\times}$, then $M(\chi) \neq 0$.

Proof. Define a ring homomorphism $c: Z G \rightarrow Z / f Z$ by $c\left(\Sigma n_{a} s_{a}\right)=$ $\sum n_{a} a \bmod f Z, n_{a} \in Z, s_{a} \in G$. For any integer $a$ prime to $f$, one has $\left(s_{a}-a\right) \omega \in f \cdot Z G$. Therefore $\omega \alpha_{\phi} \equiv \omega c\left(\alpha_{\phi}\right) \bmod f \cdot Z G$. Now $c\left(\alpha_{\phi}\right) \equiv$ $\Pi_{q}\left(1-b^{g / q}\right) \bmod f Z$, where $s_{b}=s_{\phi}$ and $g=(p-1) p^{r-1}$. The factor $1-b^{g / q}$ is prime to $p$ for $q \neq p$, and $p^{r-1}$ is the exact power of $p$ dividing $1-b^{g / q}$ when $q=p$. Thus $\omega \alpha_{\phi} \not \equiv 0 \bmod f \cdot Z G$, so $\omega \alpha_{\phi} \neq 0$; finally $M(\chi) \neq 0$ by $(3.1)$.

(3.3) Proposition. If $f=p^{r}$ and $\chi$ is an imaginary character with conductor $f$ and order a power of 2 , then $M(\chi) \neq 0$.

Proof. Since the order $g$ of $\chi$ is a power of $2, \alpha_{\phi}=2 e^{-\sigma}(\phi)$. Then $\alpha_{\phi} \omega=0$ iff

$$
s_{-1} \sigma(\phi) \omega=\sigma(\phi) \omega .
$$

Let $\theta: Z G \rightarrow Z\left[G / G_{\phi}\right]$ be the canonical projection and suppose $\gamma$ is a generator of $G / G_{\phi}$. Set $\theta(\omega)=\sum_{i=1}^{g} n(i) \gamma^{i}$. If (3.4) holds, then $n(i)=n\left(i^{*}\right)$ where $i^{*}$ is defined by $\gamma^{i^{*}}=s_{-1} \gamma^{i}$. Note $i^{*} \neq i$. Since $n(i)+n\left(i^{*}\right)=f\left|G_{\phi}\right|$,

$$
2 n(i)=f\left|G_{\phi}\right|, \quad i=1, \cdots, g \text {. }
$$

If $p$ is odd, then $G_{\phi}$ has odd order (conductor of $\chi$ is $f$ ) and (3.5) is impossible. Suppose $f=2^{r}$. We take $r \geq 3$; if $f=4$, (3.5) clearly is false. Now $G_{\phi}=\left\{s_{1}, s_{-1} s^{2^{r-3}}\right\}$. Thus $n(g)=1+(-1) 5^{2^{r-3}}$ which is not $2^{r}$ as required by (3.5). Hence $\alpha_{\phi} \omega \neq 0$.

\section{REFERENCE}

1. H. Hasse, Über die Klassenzahl abelscher Zahlkörper, Akademie-Verlag, Berlin, 1952. MR 14, 141.

DEPARTMENT OF MATHEMATICS, UNIVERSITY OF ILLINOIS, URBANA, ILLINOIS 